\title{
I n silico analysis of phytochemical compounds for optimizing the inhibitors of HMG CoA reductase
}

\author{
J. Mercy Jasmine ${ }^{1}$ and R. Vanaja ${ }^{2}$ \\ ${ }^{1}$ Institute of Biochemistry, Madras Medical College, Chennai - 600 003, Tamil Nadu, India. \\ ${ }^{2}$ Department of Biochemistry, A. C. S Medical College and Hospital, Velapanchavady, Chennai - 600 077, Tamil Nadu, India.
}

\begin{tabular}{l} 
ARTICLE INFO \\
\hline Article history: \\
Received on: $06 / 08 / 2013$ \\
Revised on: $29 / 08 / 2013$ \\
Accepted on: $15 / 09 / 2013$ \\
Available online: $30 / 09 / 2013$ \\
\hline Key words: \\
HMG CoA Reductase, \\
Molecular docking, \\
Hyperlipidemia, Statins.
\end{tabular}

\begin{abstract}
HMGCoA Reductase (HMGCR), a key enzyme in the cholesterol biosynthesis, catalyzes the conversion of 3 hydroxy-3-methylglutaryl coenzyme A (HMGCoA) into mevalonate. Thus this enzyme is the target of the cholesterol-lowering drugs known as the statins. Phytoformulation1 is a polyherbal formulation consists of the extracts of plant constituents. The present study was designed to examine the ability of the secondary metabolites of Phytoformulation 1 as an antagonist to HMGCoA reductase enzyme by in silico molecular docking. The docking analysis was carried out by Ligand Fit Accelry's Discovery studio 2.1, which allows virtual screening of database of compounds and predict the strongest binders based on various scoring functions. Fifteen ligands were docked with HMG CoA reductase receptor, out of which four compounds Dichloroacetic acid 2, 2dimethylpropyl ester, 1, 6, 10-Dodecatriene-3-ol, 3, 7, 11-trimethyl-[S-(Z)]-, Isopropyl acrylate and 3, 3Dimethylacryloyl chloride were able to form hydrogen bonds with active site of target protein. Thus it may be concluded that the secondary metabolites of Phytoformulation 1 can be an inhibitor of the HMGCR receptor that could be used to treat hyperlipidemia and further in vivo studies may be carried out to prove the same.
\end{abstract}

\section{INTRODUCTION}

Coronary heart disease (CHD) is one of the most investigated diseases in medicinal history. Cholesterol is an important component of the cell membrane and is essential for the synthesis of various important metabolites. HMGCoA Reductase (HMGCR), a key enzyme in the cholesterol biosynthesis, catalyzes the conversion of 3-hydroxy-3-methylglutaryl coenzyme A (HMGCoA) into mevalonate. Human HMGCR consists of polypeptide chains of 888 amino acids with three functional portions: Residues 1-339 span the membrane of the endoplasmic reticulum eight times; residues 340-459 connect the membrane portion to the catalytic portion (residues 460-888), which resides in the cytoplasm. This enzyme is anchored in the membrane of the endoplasmic reticulum, having seven transmembrane domains, with the active site located in a long carboxyl terminal domain in the cytosol (Nakanishi et al., 1988). The inhibition of this enzyme results in the significant decrease in cholesterol levels (Goldstein and Brown, 1990) and thus reduces the risks of stroke and mortality (Herbert et al., 1997).

\footnotetext{
* Corresponding Author

J. Mercy Jasmine, Institute of Biochemistry, Madras Medical College,

Chennai - 600 003, Tamil Nadu, India. Email: jasmine.mercy@gmail.com
}

Normally in mammalian cells this enzyme is suppressed by cholesterol derived from the internalization and degradation of low density lipoprotein (LDL) via the LDL receptor as well as oxidized species of cholesterol. Thus this enzyme is the target of the widely available cholesterol-lowering drugs known collectively as the statins (Da Silva et al., 2008).

Bioinformatics involves the organization of data generated from experiments into databases, the development of new algorithms and software, using it for the interpretation and analysis of data. In the present study, Phytoformulation 1, a polyherbal formulation was analysed by GCMS and compounds were identified. These secondary metabolites were screened with the database of available structures, and algorithms adapted from artificial intelligence applications to understand the applicability of the docking tool. The compounds thus screened with docking tools provide a preliminary data for refinement of the chemical structures or modification of the specific site on a target protein or nucleotide, enabling binding at the site that is being modelled computationally with several different techniques. The availability of the structural information aids in proper selection of target for inhibitor discovery as the binding sites of the molecules are identified (Nicola et al., 2008). 


\section{MATERIALS AND METHODS}

Bioinformatics online databases such as PubMed, Pubchem and PDB were used. PubMed database is designed to provide access to citations from biomedical journals. PubChem database provides information on the biological activities of small molecules. PubChem also provides a fast chemical structure similarity search tool. The Protein Data Bank (PDB) is a repository for the 3-D structural data of large biological molecules, such as proteins and nucleic acids.

\section{Docking}

The docking analysis of the compounds with HMGCoA reductase (HMGCR) was carried out by Ligand Fit of Accelry's Discovery studio 2.1 (Accelry's Software Inc.). The software allows us to virtually screen a database of compounds and predict the strongest binders based on various scoring functions. The collection of enzyme substrate complexes were identified via docking and their relative stabilities were evaluated using their binding affinities. LigandFit was used for accurately docking ligands into protein active sites employing a cavity detection algorithm.

A shape comparison filter is combined with a Monte Carlo conformational search for generating ligand poses consistent with the active site shape. Candidate poses are minimized in the context of the active site using a grid based method for evaluating protein-ligand interaction energies. The method appears quite promising, reproducing the $\mathrm{X}$-ray structure ligand pose within an RMSD of 2A. A high-throughput screening study applied to the HMGCoA reductase receptor is also presented in which LigandFit, when combined with LigScore, an internally developed scoring function, yields very good hit rates for a ligand pool seeded with known actives (Venkatachalam et al., 2003).

\section{Docking Protocol \\ Protein Preparation}

The crystal structure of HMG-CoA 1HWK was prepared and the active site was identified. The ligands and crystallographic water molecules were removed from the protein and the chemistry of the protein was corrected for missing hydrogen. Crystallographic disorders and unfilled valence atoms were corrected using alternate conformations and valence monitor options. Following the above steps of preparation, the protein was subjected to energy minimization using the CHARMm force field.

\section{Ligand Preparation}

The aqueous extract of the polyherbal formulation was analyzed using GCMS technique and 15 compounds were identified using NIST (National Institute of Standard and Technology). These secondary metabolites were used as the ligands. The three dimensional structures of compounds were downloaded in .sdf format from PubChem and Chemspider database. Hydrogen Bonds were added and the energy was minimized using CHARMm force field. Molecular weight, $\log P$ and number of Hydrogen-bond donors and acceptors for the active ligands were noted (Table 1).

\section{Docking Studies}

The active site of the protein was first identified and it is defined as the binding site. The binding sites were defined based on the ligands already present in the PDB file (i.e. statin binding site region) which were followed by site sphere definition. The determination of the ligand binding affinity was calculated using LigScore and PLP1, JAIN and Dock score were used to estimate the ligand-binding energies. Apart from these, other input parameters for docking were set as default options.

Table. 1: Properties of Ligands.

\begin{tabular}{|c|c|c|c|c|c|}
\hline S.No & Molecules & $\begin{array}{c}\text { Molecular Weight } \\
\text { (Daltons) }\end{array}$ & $\log P$ & $\begin{array}{l}\text { H-bond } \\
\text { Donor }\end{array}$ & $\begin{array}{c}\text { H-bond } \\
\text { Acceptor }\end{array}$ \\
\hline 1. & Isopropyl acrylate & 114.14 & 1.651 & 0 & 2 \\
\hline 2. & Hexane, 2,2,5,5-tetramethyl- & 142.28 & 5.293 & 0 & 0 \\
\hline 3. & 3,3-Dimethylacryloyl chloride & 118.56 & 1.465 & 0 & 1 \\
\hline 4. & Amyl Nitrite & 117.14 & 2.241 & 0 & 3 \\
\hline 5. & Caffeine & 194.19 & -0.628 & 0 & 6 \\
\hline 6. & 1-Methylene-2b-hydroxymethyl-3,3-dimethyl-4b-(3-methylbut-2-enyl)-cyclohexane & 222.36 & 5.201 & 1 & 1 \\
\hline 7. & Cyclohexene, 1-methyl-4-(1-methylethenyl)-, (S)- & 136.23 & 4.552 & 0 & 0 \\
\hline 8. & Octane, 2-bromo- & 192.12 & 4.71 & 0 & 0 \\
\hline 9. & 1-Penten-3-one, 4,4-dimethyl-1-phenyl- & 188.26 & 3.369 & 0 & 1 \\
\hline 10. & Nonane, 1-iodo- & 254.15 & 5.685 & 0 & 0 \\
\hline 11. & 1-Iodoundecane & 282.20 & 6.704 & 0 & 0 \\
\hline 12. & Tetradecane, 1-iodo- & 324.28 & 8.233 & 0 & 0 \\
\hline 13. & Dichloroacetic acid, 2,2-dimethylpropyl ester & 198.02 & 2.75 & 0 & 2 \\
\hline 14. & 1-Propene, 2-nitro-3-(1-cyclooctenyl) & 195.25 & 1.357 & 0 & 3 \\
\hline 15. & 1,6,10-Dodecatrien-3-ol, 3,7,11-trimethyl-, [S-(Z)]- & 222.36 & 4.682 & 1 & 1 \\
\hline
\end{tabular}

Table. 2: Structural alignment results.

\begin{tabular}{|c|c|c|c|c|c|c|c|c|}
\hline S.No & Name & Ligscore1 & Ligscore2 & - PLP1 & -PLP2 & JAIN & -PMF & Dock Score \\
\hline 1. & Dichloroacetic acid, 2,2-dimethylpropyl ester & 2.94 & 3.11 & 23.31 & 23.93 & -1.15 & 19.5 & 29.219 \\
\hline 2. & 1,6,10-Dodecatriene-3-ol,3,7,11-trimethyl-[S-(Z)]- & 2.37 & 3.99 & 47.69 & 49.75 & -1.87 & 49.0 & 41.743 \\
\hline 3. & Isopropyl acrylate & 2.77 & 3.34 & 29.07 & 27.83 & 0.76 & 23.12 & 28.169 \\
\hline 4. & 3,3-Dimethylacryloyl chloride & 1.26 & 2.57 & 19.71 & 23.34 & 1.03 & 13.09 & 15.461 \\
\hline
\end{tabular}


Table. 3: Hydrogen bond interactions between the enzyme HMG CoA Reductase and the ligands.

\begin{tabular}{|c|c|c|c|c|c|c|}
\hline S.No & Ligand & Amino acid & $\begin{array}{c}\text { Atom in amino } \\
\text { acid }\end{array}$ & Position & $\begin{array}{l}\text { Atom in } \\
\text { Ligand }\end{array}$ & $\begin{array}{c}\text { Hydrogen Bond } \\
\text { length }\left(\mathbf{A}^{\mathbf{0}}\right)\end{array}$ \\
\hline \multirow{5}{*}{1} & \multirow{5}{*}{ Dichloroacetic acid, 2, 2-dimethylpropyl ester } & LYS & $\mathrm{HZ1}$ & 502 & $\mathrm{O} 3$ & 1.71 \\
\hline & & LYS & HZ2 & 502 & $\mathrm{O} 3$ & 2.49 \\
\hline & & LYS & HZ3 & 502 & $\mathrm{O} 3$ & 1.71 \\
\hline & & LYS & HZ3 & 502 & $\mathrm{O} 4$ & 2.44 \\
\hline & & ASN & HD22 & 567 & $\mathrm{Cl} 2$ & 2.10 \\
\hline 2 & 1, 6, 10-Dodecatriene-3-ol, 3, 7, 11-trimethyl-[S-(Z)]- & LYS & HZ3 & 502 & $\mathrm{O} 1$ & 1.58 \\
\hline \multirow{3}{*}{3} & \multirow{3}{*}{ Isopropyl acrylate } & LYS & HZ1 & 502 & $\mathrm{O} 1$ & 2.30 \\
\hline & & LYS & $\mathrm{HZ2}$ & 502 & $\mathrm{O} 1$ & 2.48 \\
\hline & & LYS & $\mathrm{HZ2}$ & 502 & $\mathrm{O} 2$ & 1.63 \\
\hline 4 & 3, 3-Dimethylacryloyl chloride & LYS & HZ2 & 502 & $\mathrm{O} 2$ & 1.65 \\
\hline
\end{tabular}

\section{RESULTS AND DISCUSSION}

A large number of medicinal plants are proven to possess beneficial therapeutic potentials and act a rich source of secondary metabolites with diverse array of biological activities. These secondary metabolites may act as lead molecules. Molecular docking continues to hold great promise in the field of computer based drug design, as a result novel ligands for receptors of known structure were designed and their interaction energies were calculated using the scoring functions (Irwin et al., 2002). In recent times number of reports citing successful application of in silico studies in drug developing program in different therapeutic areas are expanding rapidly. An interesting example involves a type I TGF _ receptor kinase inhibitor. The same molecule (HTS466284/LY-364947), a $27 \mathrm{nM}$ inhibitor, was discovered independently using virtual screening by Biogen IDEC (Singh et al., 2003) and cell based high-throughput screening (Sawyer JS et al., 2003). Another in silico modelling drug development program led to clinical trials of a novel, potent, and selective anti-anxiety, anti-depression 5-HT1A agonist (Becker et al., 2006).

Top ranked ligands were usually taken for binding affinity studies. The validation process consisted of two parts, hydrogen bond details of the top-ranked docked pose and prediction of binding energy between the docked ligand and the enzyme using various score calculated using Discovery studio (LigScore2, LigScore1, PLP, PMF, and JAIN scores were taken for the analysis). In a recent study on the inhibitory studies of HMGCoA reductase molecular docking was successfully carried out by identification of the lead molecules by virtual screening against ligand data-set prepared from different literature search and ZINC database. These lead molecules were optimized using molecular dynamics and show good docking score than statins (Annabhimoju and Vuruputuri, 2013). It is estimated that docking programs currently dock $70-80 \%$ of ligands correctly (Congreve et al., 2005).

\section{Hydrogen Bond Details}

Out of the 15 ligands which were docked with HMG CoA Reductase receptor, only 4 compounds were able to form hydrogen bonds with active site of target protein. A close view of the binding interactions of HMG CoA Reductase with the compounds (i) Dichloroacetic acid 2, 2-dimethylpropyl ester, (ii) 1, 6, 10-Dodecatriene-3-ol, 3, 7, 11-trimethyl-[S-(Z)]-, (iii) Isopropyl acrylate and (iv) 3, 3-Dimethylacryloyl chloride were shown in Fig.1,2,3,4 respectively. Ligand is colored in yellow and pink (in ball and-stick drawing) where as amino acids involved in hydrogen bonding was shown in green color.

As shown in Fig. 1, there are five hydrogen bonds (shown as green dotted lines) formed between the compound Dichloroacetic acid 2, 2-dimethylpropyl ester and HMGCoA Reductase. The amino acid residues involved in forming the hydrogen bonds with the enzyme were: GLY 542, PRO 477, ASN 567, and ALA 478 (Table 3). The compound 1, 6, 10Dodecatriene-3-ol, 3, 7, 11-trimethyl-[S-(Z)] - formed one hydrogen bond with the protein (Fig. 2) and the residue involved is: ARG 495 (Table 3). Isopropyl acrylate formed three hydrogen bonds with the enzyme (Fig. 3) and here the residues involved were: ARG 495, LEU 498 (Table 3). 3, 3-Dimethylacryloyl chloride formed one hydrogen bond (Fig. 4) and the residues involved in the formation were: PRO 477, LEU 499, and ALA 478 (Table 3).

\section{Docking Score}

The study aimed to rank the docked complex through the scores was copied from the table browser view of Discovery studio for binding affinity analysis. Table 2 shows the different score values of top ranked ligands. The score values include Ligscore1\&2 (Protein- Ligand Affinity Energy) (Krammer et al., 2005), Piecewise Linear Potential (PLP) PLP1, PLP2 (Steric and $\mathrm{H}$-bonding intermolecular function, Higher PLP scores indicate stronger receptor-ligand binding (larger pKi values) (Gehlhaar et al., 1995; Gehlhaar et al., 1999), JAIN(sum of five interaction terms namely Lipophilic interactions, Polar attractive interactions, Polar repulsive interactions, Solvation of the protein and ligand, an entropy term for the ligand) ( Jain, 1996), PMF(developed based on statistical analysis of the 3D structures of protein-ligand complexes, scores are calculated by summing pairwise interaction terms over all interatomic pairs of the receptor-ligand complex. A higher score indicates a stronger receptor-ligand binding affinity) (Muegge 2006; Muegge et al., 1999) and DockScore (Candidate ligand poses are evaluated and prioritized according to the DockScore function). The determination of the ligand binding affinity was calculated using the shape-based interaction energies of the ligand with the protein. The two scoring methodologies 
namely LigScore and PLP1 were used to estimate the ligandbinding energies. Larger score value indicates better ligandbinding affinity. Based on all the dock score values (Dichloroacetic acid, 2,2-dimethylpropyl ester (Dock score=29.219), 1,6,10-Dodecatriene-3-ol,3,7,11-trimethyl-[S-(Z)](Dock Score=41.743), Isopropyl acrylate (Dock Score=28.169), 3,3-Dimethylacryloyl chloride (Dock Score $=15.461)$ ), it was predicted that the ligands 1, 6, 10-Dodecatriene-3-ol, 3, 7, 11trimethyl-[S-(Z)]- and 3, 3-Dimethylacryloyl chloride have similar and good binding affinities towards the protein. Here the maximum of five hydrogen bonds were formed between the protein and the ligand, Dichloroacetic acid, 2, 2-dimethylpropyl ester (Fig.1).

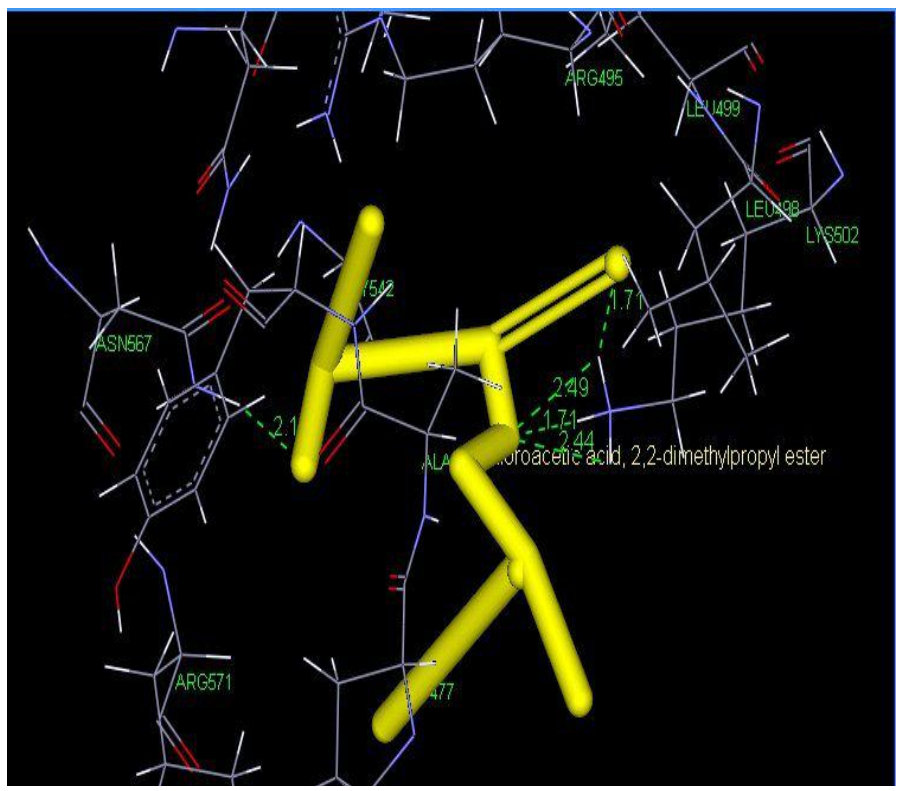

Fig. 1: Binding Interactions of HMG CoA reductase with Dichloroacetic acid, 2, 2-dimethylpropyl ester.

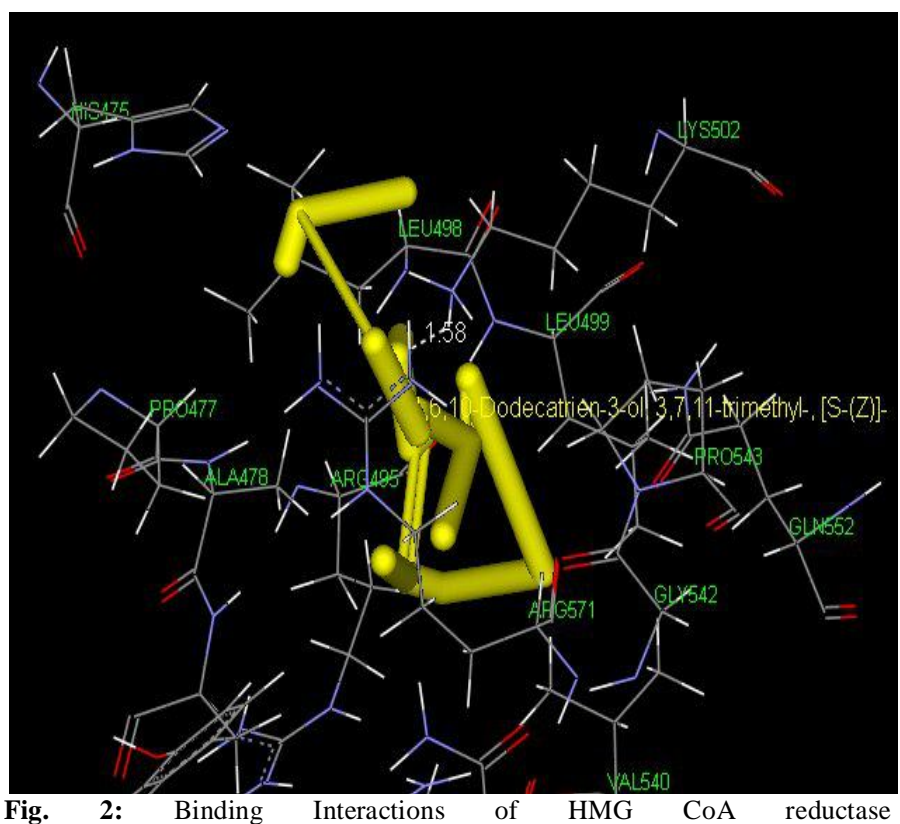
with 1, 6, 10 - Dodecatrien - 3-ol, 3, 7, 11 - trimethyl-, [S-Z)]-
Three hydrogen bonds were formed between the enzyme and the ligand Isopropyl acrylate (Fig. 3). 1, 6, 10-Dodecatriene-3-ol, 3, 7, 11-trimethyl-[S-(Z)] - (Fig. 2) and 3, 3-Dimethylacryloyl chloride (Fig.4) formed one hydrogen bond with the enzyme. Thus the concept of protein-ligand interaction helps in analyzing the binding properties of the protein HMG CoA Reductase with its inhibitors. The study also reported that the residues Lys 502 and Asn 567 (Table. 3) play an important role in binding mechanism.

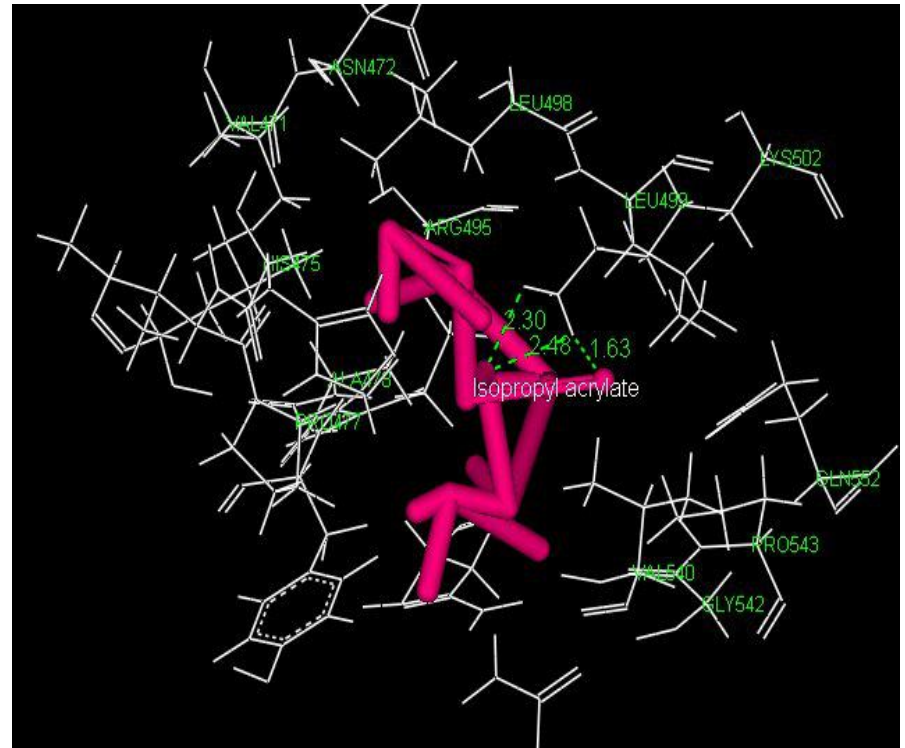

Fig. 3: Binding Interactions of HMG CoA reductase with Isopropyl acrylate.

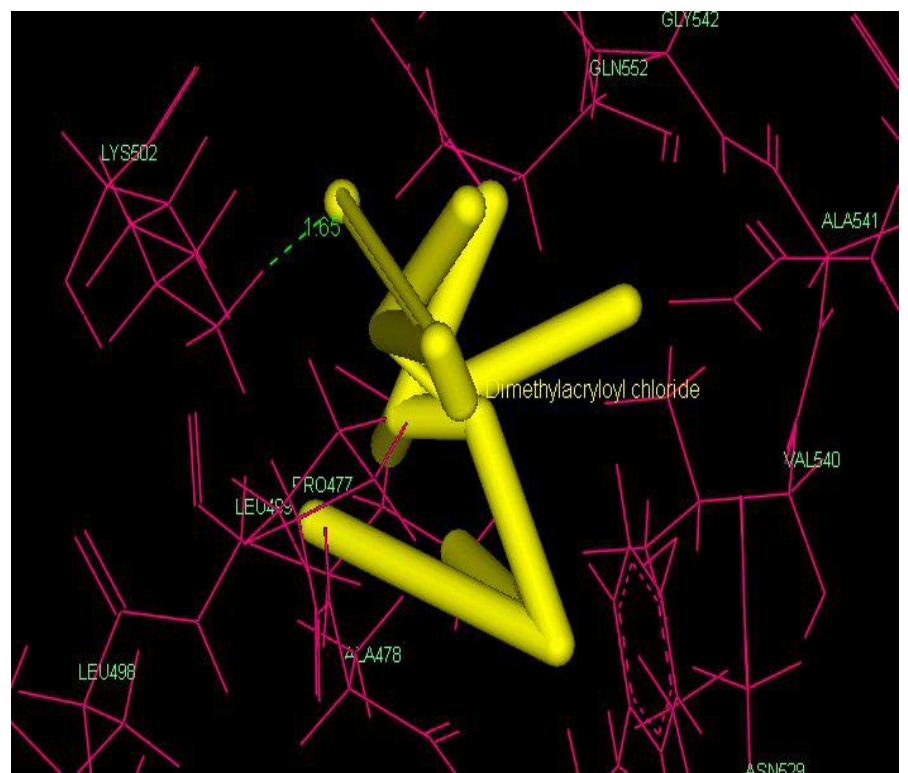

Fig. 4: Binding Interactions of HMG CoA reductase with 3,3 Dimethylacryloyl chloride.

The ligand 1, 6, 10-Dodecatriene-3-ol, 3, 7, 11-trimethyl-[S-(Z)] showed high binding activity towards the enzyme, though the number of hydrogen bond interactions are less. Hydrogen bond formation also makes important contributions to the interactions between ligand and the enzyme. The ligands Dichloroacetic acid, 2, 2-dimethylpropyl ester and 
Isopropyl acrylate formed five and three hydrogen bonds respectively. Thus based on the results obtained it may be concluded that the compounds of the Phytoformulation 1 posses antihyperlipidemic activity. Further invivo studies can be carried out to prove the efficacy of these compounds.

\section{CONCLUSION}

Understanding the interactions between proteins and ligands is crucial for the pharmaceutical and functional food industries. The emergence of bioinformatics has offered a platform to explore diseases at molecular level using computational tools. The Protein-Ligand interaction plays a significant role in structure based drug designing. The experimental structures of these protein/ligand complexes are usually obtained, by time-consuming techniques such as X-ray crystallography or NMR. These screening methods are routinely and extensively used to reduce cost and time of drug discovery. In the present work, the enzyme HMG CoA Reductase and the ligand molecules were taken to explore the binding mechanism of identified plant compounds from GC-MS study to the HMG CoA Reductase enzyme. Therefore, this study emphasizes the importance of small molecules from various plant sources and their use to enhance protein-ligand interaction studies in silico. Further work can be extended to study the receptor- ligand interactions experimentally and evaluation of their biological activity would help in specifying compounds against hyperlipidemia based on screening, docking and consensus scoring techniques.

\section{ACKNOWLEDGEMENTS}

The authors would like to acknowledge their gratification to Mr. M. Santhosh for his assistance and guidance with this paper.

\section{REFERENCES}

Becker OM, Dhanoa DS, Marantz Y, Chen D, Shacham S, Cheruku S, Heifetz A, Mohanty P, Fichman M, Sharadendu A, Nudelman $\mathrm{R}$, Kauffman M, Noiman S. An Integrated in silico 3D Model-Driven Discovery of a Novel, Potent, and Selective Amido sulfonamide 5-HT1A Agonist (PRX-00023) for the Treatment of Anxiety and Depression. J Med Chem, 2006; 49: 3116-3135.

Congreve M, Murray CW, Blundell TL. Structural biology and drug discovery. Drug Discov Today, 2005; 10: 895-907.

Da Silva V B, Taft CA, Silva CH. Use of virtual screening, flexible docking, and molecular interaction fields to design novel HMGCoA reductase inhibitors for the treatment of hypercholesterolemia. J Phys Chem, 2008; 112: 2007-2011.

Gehlhaar DK, Bouzida D, Rejto PA, Parrill L, Rami Reddy M. 1999. Series title: ACS symposium series, 719. American Chemical Society, Washington, DC. Pp. 292-311
Gehlhaar DK, Verkhivker GM, Rejto PA, Sherman CJ, Fogel DB, Fogel LJ, Freer ST. Molecular Recognition of the Inhibitor AG-1343 by HIV-1 Protease: Conformationally Flexible Docking by Evolutionary Programming. Chemistry \& Biology, 1995; 2: 317.

Goldstein JL. and Brown, MS. Regulation of the mevalonate pathway. Nature, 1990; 343: 425-430.

Hebert PR., Gaziano JM, Chan KS, Hennekens CH. Cholesterol lowering with statin drugs, risk of stroke and total mortality. An overview of randomized trials. J. Am. Med. Assoc., 1997; 278: 313-321.

Irwin JJ, Lorber DM, McGovern SL, Wei B, Shoichet BK. 2002. Computational Nanoscience and Nanotechnology.

Jain AN. Scoring noncovalent protein-ligand interactions: A continuous differentiable function tuned to compute binding affinities. J Comput Aided Mol Design, 1996; 10: 427-440.

Krammer A, Kirchhoff PD, Jiang X, Venkatachalam CM, Waldman M. LigScore: a novel scoring function for predicting binding affinities. J Mol Graph Model, 2005; 23: 395-407.

Muegge I and Martin YC. A General and Fast Scoring Function for Protein-Ligand Interactions: A Simplified Potential Approach. J Med Chem, 1999; 42: 791.

Muegge I. PMF Scoring Revisited. J Med Chem, 2006; 49: 5895-5902.

Nakanishi M, Goldstein JL, Brown MS. Multivalent control of 3- hydroxy-3-methylglutaryl coenzyme A reductase. Mevalonate-derived product inhibits translation of mRNA and accelerates degradation of enzyme. Journal of Biological Chemistry, 1988; 263: 8929-8937.

Nicola A, Smith CA, Abagyan, R. New method for the assessment of all Drug-Like Pockets Across a structural Genome. Journal of Computational Biology, 2008; 15: 231-240.

Ramakrishna Annabhimoju and Uma Vuruputuri. 159 Computational approach for optimizing inhibitors of $1 \mathrm{HWK}$ (HMG-CoA reductase). Journal of Biomolecular Structure and Dynamics, 2013; 31: 103-104.

Sawyer JS, Anderson BD, Beight DW, Campbell RM, Jones ML, Herron DK, Lampe JW, McCowan JR, McMillen WT, Mort N, Parsons S, Smith EC, Vieth M, Weir LC, Yan L, Zhang F, Yingling JM. Synthesis and activity of new aryl- and heteroaryl-substituted pyrazole inhibitors of the transforming growth factor-beta type I receptor kinase domain. J Med Chem, 2003; 46: 3953-3956.

Singh J, Chuaqui CE, Boriack-Sjodin PA, Lee WC, Pontz T, Corbley MJ, Cheung HK, Arduini RM, Mead JN, Newman MN, Papadatos JL, Bowes S, Josiah S, Ling LE. Successful shape-based virtual screening: the discovery of a potent inhibitor of the type I TGFbeta receptor kinase (TbetaRI). Bioorg Med Chem Lett, 2003; 13: 4355-4359.

Venkatachalam $\mathrm{CM}$, Jiang $\mathrm{X}$, Oldfield $\mathrm{T}$, Waldman $\mathrm{M}$. LigandFit: A Novel Method for the Shape-Directed Rapid Docking of Ligands to Protein Active Sites. J Mol Graph Modell, 2003; 21: 289-307.

\section{How to cite this article:}

J. Mercy Jasmine, R. Vanaja., In silico analysis of phytochemical compounds for optimizing the inhibitors of HMG CoA reductase. J App Pharm Sci, 2013; 3 (09): 043-047. 